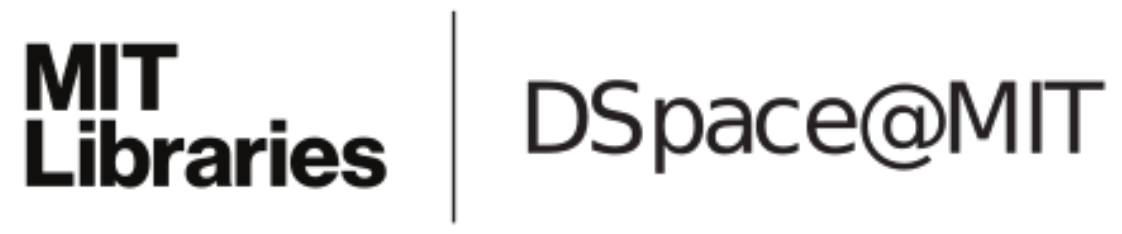

\author{
MIT Open Access Articles
}

Stability analysis for class of switched nonlinear systems

The MIT Faculty has made this article openly available. Please share how this access benefits you. Your story matters.

Citation: Shaker, H.R., and J.P. How. "Stability analysis for class of switched nonlinear systems." American Control Conference (ACC), 2010. 2010. 2517-2520. (O2010 IEEE.

Publisher: Institute of Electrical and Electronics Engineers

Persistent URL: http://hdl.handle.net/1721.1/58900

Version: Final published version: final published article, as it appeared in a journal, conference proceedings, or other formally published context

Terms of Use: Article is made available in accordance with the publisher's policy and may be subject to US copyright law. Please refer to the publisher's site for terms of use. 


\section{Stability Analysis for Class of Switched Nonlinear Systems}

\author{
Hamid Reza Shaker
}

\author{
Jonathan P. How
}

\begin{abstract}
Stability analysis for a class of switched nonlinear systems is addressed in this paper. Two linear matrix inequality (LMI) based sufficient conditions for asymptotic stability are proposed for switched nonlinear systems. These conditions are analogous counterparts for switched linear systems which are shown to be easily verifiable and suitable for design problems. The results are illustrated by numerical examples.
\end{abstract}

\section{INTRODUCTION}

$\mathrm{H}$ ybrid and switched dynamical systems received a lot of attention over the last decade due to their capability for mathematical modeling of physical systems as well as manmade systems [1]. There is a growing demand in industry for methods to model, analyze, and control hybrid and switched dynamical systems.

Among different problems in this field, a lot of research has been devoted to the study of the stability of switched and hybrid systems [1][3]. Most of the methods which have been proposed so far for stability analysis of hybrid systems are devoted to switched linear systems. The stability of switched systems under arbitrary switching signal is guaranteed by the existence of a common Lyapunov function. In the linear case, many approaches have been presented to construct common Lyapunov functions. The problem is more complicated for switched nonlinear systems and relatively fewer results have been reported in this context. The existence of a common Lyapunov function is only sufficient for the stability of switched systems and can be rather conservative. There are examples of systems that do not posses a common Lyapunov function, but are stable under arbitrary switching signals. Due to the conservatism of the methods for stability analysis which are based on the common Lyapunov function, some attention has been paid to a less conservative class of Lyapunov functions, called switched quadratic Lyapunov functions [2],[12]. In this paper, we propose sufficient stability conditions based on switched quadratic Lyapunov functions for a class of switched nonlinear systems. The conditions are similar to their counterpart in [2] for switched linear systems. These linear matrix inequalities (LMI) conditions are easy to check

H. R. Shaker is currently with Department of Aeronautics and Astronautics, Aerospace Controls Laboratory, Massachusetts Institute of Technology as a visiting researcher and also with Department of Electronic Systems, Section of Automation and Control, Aalborg University (hrshaker@mit.edu).

J. P. How is a Richard Cockburn Maclaurin Professor of Aeronautics and Astronautics, Aerospace Controls Laboratory, Massachusetts Institute of Technology, Cambridge, MA (jhow@mit.edu ) and suitable for controller synthesis.

We study the class of discrete-time switched nonlinear systems of the form:

$$
\Sigma:\left\{\begin{array}{l}
x(k+1)=A_{\sigma(k)} \Phi_{\sigma(k)}(x(k)) \\
y(k)=C_{\sigma(k)} \Phi_{\sigma(k)}(x(k))
\end{array}\right.
$$

where $x(k) \in \mathbb{R}^{n}$ is the state, $y(k) \in \mathbb{R}^{p}$ is the output and $\sigma: \mathbb{Z}^{\geq 0} \rightarrow \mathrm{K}=\{1,2, \ldots,|\mathrm{K}|\}$, is the switching signal that is a piecewise constant map of the time index. $\mathrm{K}$ is the set of discrete modes, which is assumed to be finite. For each $i \in \mathrm{K}, A_{i}, C_{i}$ are matrices of appropriate dimensions. Furthermore:

$$
\Phi_{i}(x(k)):=\left[\begin{array}{c}
\bar{\Phi}_{i}\left(x_{1}(k)\right) \\
\bar{\Phi}_{i}\left(x_{2}(k)\right) \\
\vdots \\
\bar{\Phi}_{i}\left(x_{n}(k)\right)
\end{array}\right]
$$

where:

$$
\bar{\Phi}_{i} \in O L:=\{\phi: \mathbb{R} \rightarrow \mathbb{R}|\forall s, t \in \mathbb{R},| \phi(s)+\phi(t)|\leq| s+t \mid\} .
$$

It is worth mentioning that subsystems with the above description are from the class of so-called $\Phi$-systems [4]. In some literature, they have been called $\sigma$-systems[6][7]. It is clear from the description that the nonlinearity of this class of systems is odd and 1-Lipschitz. The standard saturation and the hyperbolic tangent (popular activation function in neural network) are examples of this type of nonlinear systems[4][7]-[10]. The discrete-time recurrent artificial neural network is a special case of $\Phi$-systems [7][9]. Furthermore, results related to this class of nonlinear systems have potential applications in the classical problems related to uncertain nonlinearities such as Lur'e systems[11].

The notation used in this paper is as follows: $M^{*}$ denotes transpose of matrix if $M \in \mathbb{R}^{n \times m}$ and complex conjugate transpose if $M \in \mathbb{C}^{n \times m}$. The standard notation $>, \geq(<, \leq)$ is used to denote the positive (negative) definite and semidefinite ordering of matrices.

\section{Positive Diagonal Dominant Matrices}

In this section we recall a definition and results which we will use in the sequel.

Definition 1: A matrix $P$ is said to be positive diagonally dominant (pdd) if: 


$$
\left\{\begin{array}{l}
P>0 \\
\left|p_{i i}\right|>\sum_{j \neq i}\left|p_{i j}\right|, \quad \forall i .
\end{array}\right.
$$

This definition simply says that a matrix is pdd if it is positive definite and row diagonally dominant.

Lemma 1 [4]: $P$ is pdd if and only if $P>0$ and there exists a symmetric $R=\left[r_{i j}\right]$ such that:

$$
\left\{\begin{array}{lr}
r_{i j} \geq 0, \quad p_{i j}+r_{i j} \geq 0, & \forall i \neq j, \\
p_{i i} \geq \sum_{j \neq i}\left(p_{i j}+2 r_{i j}\right) & \forall i .
\end{array}\right.
$$

Lemma 2 [4]: $P$ is pdd if and only if:

$$
\begin{gathered}
P>0 \\
\forall \Phi \in O L, \quad \forall \gamma \in \mathbb{R}^{n}, \quad \Phi(\gamma)^{*} P \Phi(\gamma) \leq \gamma^{*} P \gamma .
\end{gathered}
$$

Lemma 2 shows the elegant property of the pdd matrices which is useful for finding conditions for quadratic stability of $\Phi$-systems.

\section{Stability OF SWITCHED $\Phi$ - Systems}

Consider the family of the switched $\Phi$-systems described in (1). This class of systems can also be represented as:

$$
\Sigma:\left\{\begin{array}{l}
x(k+1)=\sum_{i=1}^{|K|} \zeta_{i}(k) A_{i} \Phi_{i}(x(k)) \\
y(k)=\sum_{i=1}^{|K|} \zeta_{i}(k) C_{i} \Phi_{i}(x(k))
\end{array}\right.
$$

where:

$\zeta_{i}(k)$ is the indicator function which is defined as:

$\zeta_{i}(k)= \begin{cases}1, & \text { when the switched system is described } \\ & \text { by the } i^{t h} \text { mode matrices }\left(\mathrm{A}_{i}, \mathrm{C}_{i}\right) \text { and } \Phi_{i} \\ 0, & \text { otherwise }\end{cases}$

A sufficient condition for stability is the following:

Proposition 1. The switched system (6) is asymptotically stable under an arbitrary switching signal if there exist $|K|$ symmetric pdd matrices, $P_{1}, P_{2}, \ldots, P_{|K|}$ satisfying:

$$
\left[\begin{array}{cc}
P_{i} & A_{i}^{*} P_{j} \\
P_{j} A_{i} & P_{j}
\end{array}\right]>0 \quad \forall(i, j) \in K \times K,
$$

\section{Proof:}

Let:

$$
\begin{aligned}
& V(k, \gamma(k)):=\gamma(k)^{*}\left(\sum_{i=1}^{|K|} \zeta_{i}(k) P_{i}\right) \gamma(k), \quad \gamma(k) \in \mathbb{R}^{n} \\
& \zeta(k)=\left[\begin{array}{llll}
\zeta_{1}(k) & \zeta_{2}(k) & \cdots & \zeta_{|K|}(k)
\end{array}\right],
\end{aligned}
$$

$$
\begin{aligned}
& A(\zeta(k))=\sum_{i=1}^{|K|} \zeta_{i}(k) A_{i}, P(\zeta(k))=\sum_{i=1}^{|K|} \zeta_{i}(k) P_{i} \\
& \Phi_{\sigma}(x(k))=\sum_{i=1}^{|K|} \zeta_{i}(k) \Phi_{i}(x(k))
\end{aligned}
$$

then:

$$
\begin{aligned}
& \Delta V(k, x(k))=V(k+1, x(k+1))-V(k, x(k)) \\
&=x(k+1)^{*} P(\zeta(k+1)) x(k+1)-x(k)^{*} P(\zeta(k)) x(k) \\
&=\Phi_{\sigma}^{*}(x(k)) A^{*}(\zeta(k)) P(\zeta(k+1)) A(\zeta(k)) \Phi_{\sigma}(x(k)) \\
&-x(k)^{*} P(\zeta(k)) x(k) \\
&= \Phi_{\sigma}^{*}(x(k))\left[A^{*}(\zeta(k)) P(\zeta(k+1)) A(\zeta(k))-P(\zeta(k))\right] \Phi_{\sigma}(x(k)) \\
&+\Phi_{\sigma}^{*}(x(k)) P(\zeta(k)) \Phi_{\sigma}(x(k))-x(k)^{*} P(\zeta(k)) x(k)
\end{aligned}
$$

On the other hand, since:

$P_{1}, P_{2}, \ldots, P_{|K|}$ are all pdd and $\Phi_{\sigma} \in O L$ we have from Lemma 2 that:

$$
\Phi_{\sigma}^{*}(x(k)) P(\zeta(k)) \Phi_{\sigma}(x(k))-x(k)^{*} P(\zeta(k)) x(k) \leq 0
$$

The Schur complement of (8):

$$
\left(A_{i}^{*} P_{j}\right) P_{j}^{-1}\left(P_{j} A_{i}\right)-P_{i}=A_{i}^{*} P_{j} A_{i}-P_{i}>0 \text {, }
$$

shows that:

$$
A^{*}(\zeta(k)) P(\zeta(k+1)) A(\zeta(k))-P(\zeta(k))<0
$$

Therefore:

$$
\Delta V(k, x(k))<0,
$$

which proves the stability of the switched system(6).

Note that the switched quadratic Lyapunov function is a common Lyapunov function when $P_{1}=P_{2}=\ldots=P_{|K|}$.

Therefore, the stability condition based on the switched quadratic Lyapunov function generalizes the approaches based on the common Lyapunov function and is usually less conservative.

The next proposition is similar to Proposition 1. In the stability condition of Proposition 2, we have slack variables which makes the proposition more suitable for design problems.

Proposition2. The switched system (6) is asymptotically stable under an arbitrary switching signal if there exist $|K|$ symmetric positive diagonal matrices, $S_{1}, S_{2}, \ldots, S_{|K|}$ and $|K|$ matrices, $G_{1}, G_{2}, \ldots, G_{|K|}$,satisfying:

$$
\left[\begin{array}{cc}
G_{i}+G_{i}^{*}-S_{i} & G_{i}^{*} A_{i}^{*} \\
A_{i} G_{i} & S_{j}
\end{array}\right]>0 \quad \forall(i, j) \in K \times K
$$

\section{Proof:}

From (10), we have: $G_{i}+G_{i}^{*}-S_{i}>0$. Since $S_{i}$ is a positive diagonal matrix, $S_{i}^{-1}$ is also the positive diagonal, which implies that: $\left(G_{i}-S_{i}\right)^{*} S_{i}^{-1}\left(G_{i}-S_{i}\right) \geq 0$. Moreover:

$$
\left(G_{i}-S_{i}\right)^{*} S_{i}^{-1}\left(G_{i}-S_{i}\right)=G_{i}^{*} S_{i}^{-1} G_{i}-G_{i}^{*}-G_{i}+S_{i} .
$$

Hence: 


$$
\left[\begin{array}{cc}
G_{i}^{*} S_{i}^{-1} G_{i} & G_{i}^{*} A_{i}^{*} \\
A_{i} G_{i} & S_{j}
\end{array}\right]>0 \quad \forall(i, j) \in K \times K
$$

On the other hand:

$$
\left[\begin{array}{cc}
G_{i}^{*} S_{i}^{-1} G_{i} & G_{i}^{*} A_{i}^{*} \\
A_{i} G_{i} & S_{j}
\end{array}\right]=\left[\begin{array}{cc}
G_{i}^{*} & 0 \\
0 & S_{j}
\end{array}\right]\left[\begin{array}{cc}
S_{i}^{-1} & A_{i}^{*} S_{j}^{-1} \\
S_{j}^{-1} A_{i} & S_{j}^{-1}
\end{array}\right]\left[\begin{array}{cc}
G_{i} & 0 \\
0 & S_{j}
\end{array}\right]
$$

Therefore:

$$
\left[\begin{array}{cc}
S_{i}^{-1} & A_{i}^{*} S_{j}^{-1} \\
S_{j}^{-1} A_{i} & S_{j}^{-1}
\end{array}\right]>0
$$

where $S_{i}^{-1}$ and $S_{j}^{-1}$ are positive diagonal. $S_{i}^{-1}$ and $S_{j}^{-1}$ are obviously pdd matrices. From Proposition 1, we conclude that the switched system (6) is stable.

In Proposition 2, $S_{1}, S_{2}, \ldots, S_{|K|}$ matrices in general do not have to be diagonal. The only restriction is that the inverse of these matrices need to be pdd.

Note that specifying a matrix to be pdd is LMI (Lemma 1) and therefore to check the proposed conditions, we need to solve an LMI.

\section{NUMERICAL EXAMPLES}

In this section the proposed method is applied to two numerical examples: one is a second order switched $\Phi$ system and the other one is a third order switched system.

\section{A. Second Order Switched $\Phi$-system:}

Consider a bimodal switched $\Phi$-system with the system matrices:

$$
A_{1}=\left[\begin{array}{cc}
-0.3099 & -0.6063 \\
-0.6063 & 0.3684
\end{array}\right], A_{2}=\left[\begin{array}{cc}
-0.04904 & -0.02485 \\
0.02485 & -0.04904
\end{array}\right] .
$$

The LMI condition (8) is feasible with the solution:

with

$$
P_{1}=\left[\begin{array}{cc}
34.5791 & -0.5189 \\
-0.5189 & 35.0735
\end{array}\right], P_{2}=\left[\begin{array}{cc}
26.3119 & 0.0760 \\
0.0760 & 26.2510
\end{array}\right],
$$

$$
R_{1}=\left[\begin{array}{cc}
0 & 9.4905 \\
9.4905 & 0
\end{array}\right], R_{2}=\left[\begin{array}{cc}
0 & 5.7751 \\
5.7751 & 0
\end{array}\right] .
$$

Therefore, the switched $\Phi$-system is stable. The inverse of a two-dimensional pdd matrix is always a pdd matrix. This is not always true for higher dimensions. Because of the fact that the inverse of a two-dimensional pdd matrix is always a pdd, the following $S_{1}$ and $S_{2}$ satisfy (10).

$$
S_{1}=\left[\begin{array}{ll}
0.0289 & 0.0004 \\
0.0004 & 0.0285
\end{array}\right] S_{2}=\left[\begin{array}{ll}
0.0380 & -0.0001 \\
-0.0001 & 0.0381
\end{array}\right] \text {. }
$$

Proposition 2 for second order systems is less conservative than higher order systems and $S_{1}, S_{2}, \ldots, S_{|K|}$ do not have to be diagonal matrices.

\section{B. Third Order Switched $\Phi$-system:}

Consider a third order $\Phi$-system with the system matrices:

$A_{1}=\left[\begin{array}{ccc}-0.06515 & -0.4744 & 0.3041 \\ -0.4744 & 0.4872 & 0.3732 \\ 0.3041 & 0.3732 & -0.1271\end{array}\right], A_{2}=\left[\begin{array}{ccc}0.04419 & 0.3155 & -0.04247 \\ 0.1451 & -0.04931 & -0.2805 \\ 0.2833 & -0.01418 & 0.1554\end{array}\right]$

The LMI condition (8) is feasible for this example with the solution:

$$
\begin{aligned}
& P_{1}=\left[\begin{array}{ccc}
16.5100 & -0.4733 & -1.7526 \\
-0.4733 & 16.1032 & -0.4243 \\
-1.7526 & -0.4243 & 13.3561
\end{array}\right], P_{2}=\left[\begin{array}{ccc}
14.6794 & 0.1369 & 0.1619 \\
0.1369 & 11.8281 & -0.1509 \\
0.1619 & -0.1509 & 12.0436
\end{array}\right], \\
& R_{1}=\left[\begin{array}{ccc}
0 & 2.5952 & 3.2349 \\
2.5952 & 0 & 2.5707 \\
3.2349 & 2.5707 & 0
\end{array}\right], R_{2}=\left[\begin{array}{ccc}
0 & 2.0286 & 2.0161 \\
2.0286 & 0 & 2.1725 \\
2.0161 & 2.1725 & 0
\end{array}\right] .
\end{aligned}
$$

The switched system is stable under arbitrary switching signals.

LMI condition (10) is also feasible with the solution:

$$
\begin{aligned}
& S_{1}=\left[\begin{array}{ccc}
15.4911 & 0 & 0 \\
0 & 13.4929 & 0 \\
0 & 0 & 16.0713
\end{array}\right], S_{2}=\left[\begin{array}{ccc}
17.2202 & 0 & 0 \\
0 & 17.2319 & 0 \\
0 & 0 & 17.2063
\end{array}\right] \\
& G_{1}=\left[\begin{array}{ccc}
14.7675 & 0.4846 & 1.5626 \\
0.4846 & 12.2697 & 0.1082 \\
1.5626 & 0.1082 & 15.4927
\end{array}\right], G_{2}=\left[\begin{array}{rrr}
16.9289 & -0.0223 & -0.0117 \\
-0.0223 & 16.9435 & 0.0143 \\
-0.0117 & 0.0143 & 16.9115
\end{array}\right]
\end{aligned}
$$

Therefore Proposition 2 confirms the stability of the switched system under the arbitrary switching signals.

\section{CONCLUSION}

Two LMI-based sufficient conditions for stability analysis of a class of switched nonlinear systems are proposed. These conditions are extensions of the LMI-based stability conditions for switched linear systems to switched $\Phi$ systems. The proposed stability results are based on the switched quadratic Lyapunov functions which are usually less conservative than their counterparts which are based on common Lyapunov functions. These results can be used for controller design problems as well as model reduction of switched nonlinear systems.

\section{REFERENCES}

[1] D. Liberzon. Switching in Systems and Control. Boston, MA: Birkhauser, 2003.

[2] J. Daafouz, P. Riedinger, and C. Iung, "Stability analysis and control synthesis for switched systems: A switched Lyapunov function approach," IEEE Trans. Autom. Control, vol. 47, no. 11, pp. 18831887, Nov. 2002.

[3] H. Lin, P.J. Antsaklis, "Stability and Stabilizability of Switched Linear Systems: A Survey of Recent Results" IEEE Trans. Autom. Control, vol. 54, no. 2, pp. 308-322, Feb.2009.

[4] Chu, Y. and Glover, K., Bounds of the induced norm and model reduction errors for systems with repeated scalar nonlinearities. IEEE Trans. Automa. Control. vol.44 ,no. 2,pp.471-483,Mar. 1999.

[5] F. Albertini and E. D. Sontag ,"Uniqueness of weights for recurrentnets," in Proc. Int. Symp. Math. Theory of Networks Syst., Regensburg, Germany, Aug. 1993, vol. II, pp. 599-602.

[6] "F. Albertini and E. D. Sontag," "State observability in recurrent neural networks," Syst. Contr.Lett., vol. 22, no. 4, pp. 235-244, 1994. 
[7] E. D. Sontag "Recurrent neural networks: Some systems-theoretic aspects" in Dealing with Complexity: A Neural Network Approach, M. Karny, K. Warwick, and V. Kurkova, Eds. London, U.K.: Springer-Verlag, 1997.

[8] E. Kaszkurewicz and A. Bhaya, "On a class of globally stable neural circuits," IEEE Trans. Circuits and Syst.-I: Fundamental Theory and Appl., vol. 41, pp.171-174, Feb. 1994.

[9] N. Barabanov and D. Prokhorov, "Stability analysis of discrete time recurrent neural networks," IEEE Trans. Neural Networks, vol. 13, pp.292-303, Mar. 2002.

[10] D. Liu and A. N. Michel, Dynamical Systems with Saturation Nonlinearities: Analysis and Design, Lecture Notes in Control and Information Sciences, vol. 195. London, U.K.: Springer-Verlag, 1994.

[11] Q. Han, A. Xue, S. Liu, and X. Yu, "Robust absolute stability criteria for uncertain Lur'e systems of neutral type," International Journal of Robust and Nonlinear Control, vol. 18, pp. 278-295, 2008.

[12] L. Fang, H. Lin, and P. J. Antsaklis. Stabilization and performance analysis for a class of switched systems. In Proc. 43rd IEEE Conf. Decision Control, pages 3265-3270, 2004. 